\title{
Gestão da Atenção Primária: uma proposta de avaliação de impacto da APS em duas cidades do Nordeste do Brasil
}

\section{Galba Freire Moita}

\section{RESUMO}

Esse estudo apresenta uma proposta de avaliação de impacto na APS a partir da análise contextual da atenção primaria em duas grandes cidades do Ceará, visto que Fortaleza (gestão incremental por OSs) e Sobral (Gestão Pública) representam ambientes privilegiados para a aplicação de modelos avaliativos visando mensurar o impacto, a eficiência, a efetividade e a resolutividade aplicáveis em diferentes contextos da APS do Brasil.

Palavras-chave: Direção e Governança do Setor de Saúde, Avaliação de Processos e Resultados (cuidado de saúde), Avaliação em Saúde, Qualidade, Acesso e Avaliação da Assistência à Saúde, Avaliação do Impacto na Saúde.

\section{ABSTRACT}

This study presents a proposal for impact assessment on PHC from the contextual analysis of primary care in two large cities in Ceará, since Fortaleza (incremental management by OSs - non-profit health organization) and Sobral (Public Management) represent privileged environments for the application of evaluation models aiming to measure the impact, efficiency, effectiveness and resoluteness applicable in different contexts of PHC in Brazil.

Keywords: Health Sector Stewardship and Governance. Process and Results Evaluation (health care). Health Assessment. Quality, Access and Evaluation of Health Care. Health Impact Assessment.
Revista da Rede APS 2021

Publicada em: 20/09/2021

DOI:10.14295/aps.v3i2.210

Galba Freire Moita

(Centre for Business and

Economics Research (CeBER), Faculdade de Economia, Universidade de Coimbra, Coimbra, Portugal)

(Fundação Oswaldo Cruz, Ministério da Saúde do Brasil)

Correspondência para:

Galba Freire Moita galba.moita@saude.gov.br 


\section{INTRODUÇÃO}

A compreensão de que uma atenção primária à saúde (APS) fortalecida e adequadamente estruturada é fundamental na organização dos sistemas de saúde (Starfield, 2002). Em 1994, a Estratégia Saúde da Família (ESF) (anteriormente Programa Saúde da Família PSF) (Brasil, 1997, 2000, 2006). foi instituída como a forma organizativa da atenção primária no Brasil, visando substituir práticas tradicionais hospitalocêntricas e fortalecer ações de promoção, proteção e recuperação da saúde dos indivíduos e da família, de forma integral e contínua, tendo como locus de seu trabalho as Unidades Básicas de Saúde (UBS) e o território adscrito a estas (Almeida \& Giovanella, 2008b; Giovanella \& Mendonça, 2012; Giovanella et al., 2012). No Ceará a experiência do Programa de Saúde da Família (PSF) já acontecia em alguns municípios desde 1993 (Avila, 2011).

Neste sentido, diversos avanços no quadro de saúde do Brasil tem sido relacionados a expansão da ESF, como redução de internações hospitalares por condições sensíveis à atenção primária em crianças (Victora, 2011) e em adultos (Barreto et al., 2011), a redução de consultas não-urgentes nas emergências (Stein et al., 2002) e a redução da mortalidade infantil (Dourado et al., 2011; Macinko et al., 2007; Rasella et al., 2010), além da ampliação de controle das doenças cardiovasculares (Barreto et al., 2014; Rasella et al., 2014).

O modelo de financiamento em saúde ao longo dos anos tem sido utilizado como ferramentas capazes de auxiliar na melhoria de saúde das populações, uma vez que quanto mais investimento e/ou não serve para ajudar os gestores no planejamento e na avaliação em saúde (Arretche, 2003; Santana \& Costa, 2008; Santana, 2011; Vieira \& Zucchi, 2013), além de estabelecer os níveis de atenção à saúde. E como resultado da obtenção desse cuidado, a representação e a implementação de um adequado sistema de financiamento que são essenciais na busca de cobertura universal (Brasil, 2009, 2016).
Nesse escopo, pesquisas que avaliem regimes distintos de atendimento com diferentes modelos de financiamento e de gestão da APS em contextos comparáveis são importantes. No entanto, são incipientes os instrumentos de avaliação de no âmbito do SUS, inclusive na APS (Albuquerque \& Martins, 2017; Almeida \& Giovanella, 2008a; Moita et al., 2019; Volpato, 2014; Volpato et al., 2010). Assim, justifica-se a importância deste estudo avaliativo, pois a avaliação é vista não só como instrumento para verificar a efetividade de programas, mas utilizada também com o intuito de melhorar a qualidade dos serviços por um processo de julgamento de valor orientado para a tomada de decisão e governança (Moita, 2021; Raposo, 2007).

Uma análise de impacto adequada requer a caracterização de contexto e a análise comparativa. Assim, Fortaleza é uma das capitais brasileiras com razoável cobertura da ESF, que teve modesta ampliação da cobertura de ESF (<50\%, em 2006 e atingiu cerca de $62 \%$, em 2019), na qual optou-por modificações incrementais no regime de administração das UAPS para o modelo de gestão por OSs. Por sua vez, Sobral apresenta altos níveis de coberturas (94,19\%, em 2000 e manteve $100 \%$, desde 2013 ) com o atendimento da ESF gerenciado pelo modelo de administração direta em todas os Centros de Saúde da Família (CSF).

Portanto, Fortaleza (gestão incremental por OSs) e Sobral (Gestão Pública) representam ambientes privilegiados para a aplicação de modelos avaliativos e decisão na organização do atendimento da APS quanto ao financiamento, modelos de gestão e execução pública e/ou público-privada da APS.

No entanto, não há registro de avaliação de impactos resultantes nessas decisões. 0 objetivo desse estudo é propor um modelo avaliativo quanto ao impacto, eficiência, efetividade e resolutividade de alguns regimes de financiamento e modelos gestão da APS, instrumentalizando a decisão nos três níveis de gestão do SUS. Para tal, pretende-se mapear e analisar indicadores explicativos, por exemplo quanto as condições de saúde sensíveis a 
atenção primária (Alfradique et al., 2009), dentre outros sugeridos nesse trabalho.

\section{METODOLOGIA}

Para a realização da pesquisa optou-se, fundamentando-se em (Yin, 2010), (Bogdan \& Biklen, 2010), (Flick, 2009) e (Thomas \& Nelson, 2002), por um estudo do tipo estudo de caso, na modalidade multicaso.

O estudo é do tipo qualitativa utilizando a metodologia descritiva por um relato de experiência no processo de implantação de OSs em uma capital do Nordeste brasileiro comparadamente a outra cidade que manteve o modelo tradicional. Este relato de experiência de prática profissional será demonstrado a partir da construção legal, análise documental e dos noticiários publicados pela imprensa local durante o processo.

\section{RESULTADOS E DISCUSSÃO}

Dividiu-se essa sessão em 4 eixos: Análise contextual, o gerencialismo e as OSs no Ceará, Evolução da avaliação da APS e avaliação de impacto na APS.

\section{Análise contextual}

A descentralização da gestão do SUS avança com a transferência de responsabilidades assistenciais e de recursos financeiros, da esfera de governo federal para municipal, especialmente, pela implementação da atenção primária saúde (APS), por exemplo, pela criação e expansão da Estratégia Saúde da Família (ESF) (anteriormente Programa Saúde da Família PSF) (Brasil, 1997, 2000, 2006).

Por sua vez, a evolução da APS no Ceará está relacionada a experiência do Programa Agentes de Saúde (PAS). Entre 1979 e 1986, o trabalho dos agentes de saúde no Ceará já existia no município de Jucás (CE) e, posteriormente, ampliado para os demais municípios do estado. Em 1987, o trabalho dos agentes de saúde é oficializado com a participação de seis mil mulheres do sertão no Programa de Emergência, criado em decorrência da seca (Avila, 2011). Com o término do Programa de Emergência em agosto de 1988, foi institucionalizado o Programa Agentes de Saúde (PAS), criado pelo Decreto $n$ ㅇ 19.945 , de janeiro de 1989, posteriormente, regulamentado pela Portaria no 232/92 (Ceará, 1992) e pelo Decreto no 23.079, de fevereiro de 1994 .

Entre 1994 e 2010, as equipes de ESF aumentaram de 4.000 para mais de 31.600 , com a cobertura de mais de $50 \%$ da população brasileira (Gragnolati et al., 2013). Após 2017, foi implementada a nova Política Nacional de Atenção Básica (Nova PNAB) e anunciada a expansão das equipes de ESF, para alcance de $68 \%$ de cobertura da população brasileira. Se projetou ampliar mais 6.431 novas equipes ESF distribuídas em todos os estados do País, ao custo de $\mathrm{R} \$ 771,2$ milhões anuais para o custeio dos serviços implementados (Saúde, 2017).

No Ceará, com dados do SISAB/DAB/MS, o relatório Panorama Estadual da Atenção Primária à Saúde (Ceará, 2019b), assumindo o parâmetro de cobertura de 3.450 habitantes por equipe ESF (EqSF) traçou um mapeamento da realidade da APS por macrorregião de saúde, sendo 2.581 EqSF credenciadas e 2.387 EqSF implantadas (80\%), além de 295 EqNASF-AB credenciadas e 287 EqNASF-AB implantadas (97\%).

Por seu turno, a evolução da APS do município de Fortaleza, no período de 2006 a 2012, a ESF foi implementada sob a égide dos conceitos da Política Nacional da Atenção Básica - PNAB (Brasil, 2006, 2011), mas ainda incipiente, como na maioria das capitais do Brasil. Em recentes relatórios de Fortaleza (Fortaleza, 2020: 18) relatou a expansão de $27 \%$ do número de equipes EqESF (2013-2019), com atingimento de $62 \%$ da cobertura populacional da ESF. Além disso, em 2018, se implantou 26 Núcleos Ampliado de Saúde da Família (NASF) (Fortaleza, 2019:72). 
Outro município de grande porte do Ceará (IBGE) que tem potencial de estudo comparativo é Sobral-Ceará, que iniciou a consolidação do SUS e da APS organizando sua atenção primária com base na ESF (Andrade \& Martins Júnior, 1999). Desde 2013, atingiu $100 \%$ de cobertura da população de Sobral (Ceará, 2019a), contando atualmente com 37 Centros de Saúde da Família (CSF) (Ribeiro \& Cunha, 2018).

\section{O gerencialismo e as OSs no Ceará}

Em 1995, o Plano Diretor da Reforma do Aparelho do Estado (PDRAE) iniciou a reforma pública brasileira. Esse modelo gerencial voltada para os resultados estabeleceu três instituições administrativas base, as agências reguladoras, as agências executivas e as Organizações Sociais (OS) (Brasil, 1995).
Os processos de qualificação de Organizações Sociais (OS) no Brasil advém da era Fernando Henrique Cardoso através Lei no 9.367, de 15 de maio de 1998. O Modelo de gestão por OSS tem se disseminado em 23 estados brasileiros, o Distrito Federal e mais de 200 municípios e gerenciam mais de 800 unidades de saúde, com mais de 15 mil leitos (IBROS) (Macedo, 2018).

No Ceará, a qualificação de OSs foi regulamentada pela Lei no 12.781/1997 (sucedida pela Lei no 14.158/2008), e, dentre outras OSs levou a criação e qualificação do Instituto de Saúde e Gestão Hospitalar (ISGH) (Viana, 2010). A figura 1, mostra a evolução dos valores gastos pela Secretaria Estadual de Saúde com contratos da OSs ISGH, que mostra a magnitude e o incremento contínuo dos gastos.

Figura 1 - Evolução de gastos com OSs na Secretaria da Saúde do Ceará (2013-2019)

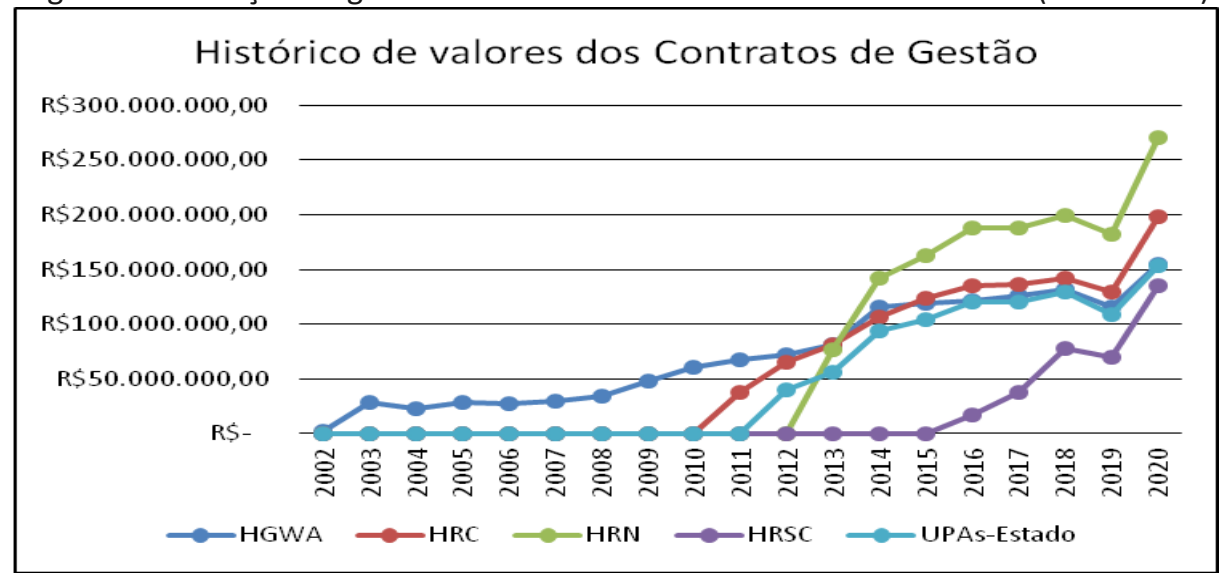

Fonte: Adaptado de (https://www.seplag.ce.gov.br/planejamento/contrato-de-gestao/)

Por sua vez, na saúde de Fortaleza os problemas sobre gestão por OSs aparentemente são relatados ao menos desde julho de 2009, em contratos de gestão em saúde superiores a $\mathrm{R} \$$ 5,5 milhões/anuais e cinco mil funcionários terceirizados geridos pela OSs denominada Instituto de Desenvolvimento Tecnológico e Apoio à Gestão e Saúde (IDGS), que segundo foi noticiado se encerrou o convênio com o IDGS, em junho de 2013 (De Lima, 2013). Em 2018, foi aprovada a Lei no 513/2018 (Cabral, 2019), sobre a qualificação de Organizações Sociais
(OSs), que flexibilizou os artigos 2 e 3 da lei 10.029/2013 (sucedânea da lei no 8.704/2003).

Nesse período houve um acirramento das disputas dentro do Conselho Municipal de Saúde de Fortaleza, relatados por Moita et al. (2021), nos debates nas exigências de controle e acompanhamento e dos estudos que gerem evidências científicas das vantagens da terceirização das unidades, conforme acórdãos do TCU (acórdãos no 3.239/2013, no 352/2016, no 1.215/2016 e no 2.444/2016). Mesmo assim, a figura 2 mostra os avanços do modelo, com 
repasse financeiro que ultrapassou $\mathrm{R} \$ 252$ milhões em 2019.

Figura 2 - Evolução de gastos com OSs na Secretaria da Saúde de Fortaleza (2013-2019).

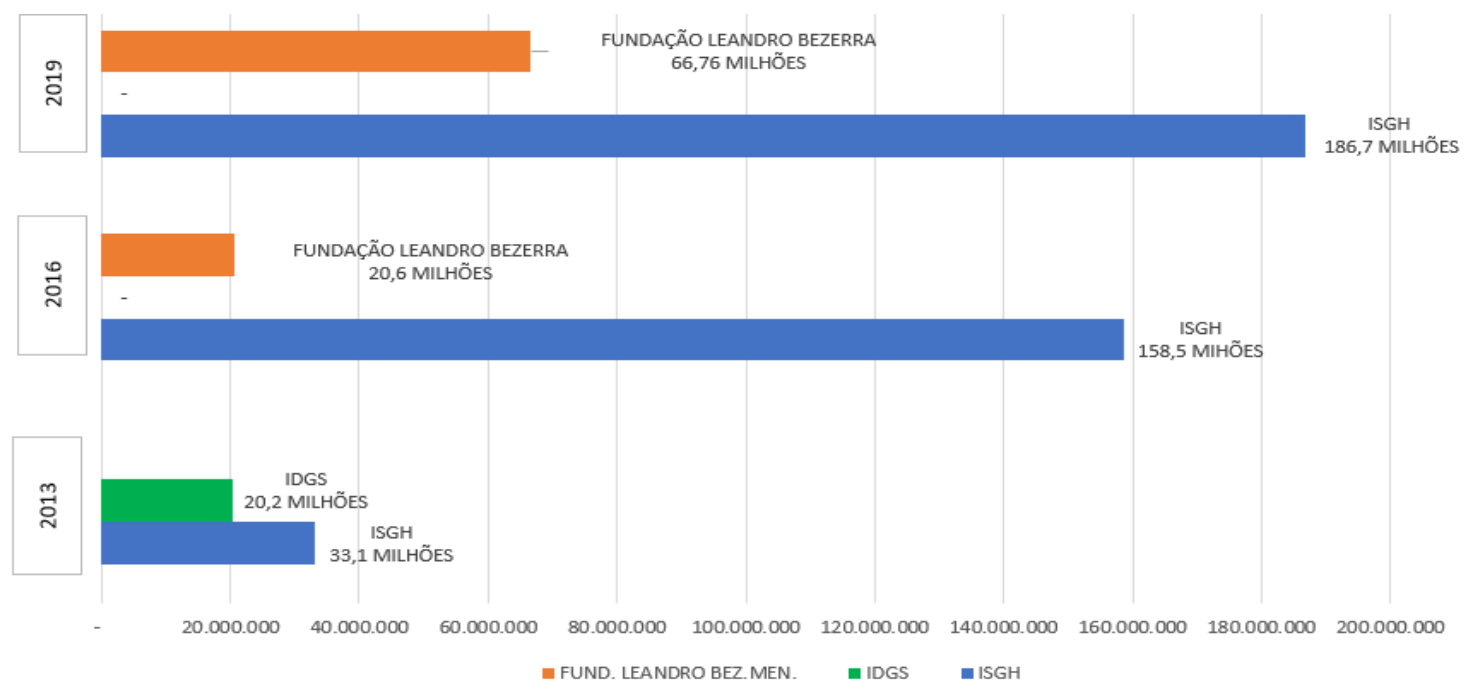

Fonte: Adaptado de (https://www.tce.ce.gov.br/portal)

\section{Evolução da avaliação da APS}

Nos últimos anos, a avaliação em saúde configurou-se como um importante instrumento para o planejamento e a gestão de sistemas e de serviços em saúde (Sancho \& Dain, 2012), tendo diversas abordagens metodológicas (Bosi \& Uchimura, 2007).

Por sua vez, Starfield (2002) destaca que a importância de medir os atributos da APS visando o impacto da APS na saúde da população. Identificou quatro atributos essenciais para avaliação da APS: acesso de primeiro contato, longitudinalidade, integralidade e coordenação. A capacidade de interação dos serviços com os indivíduos e com a comunidade foram nominados de atributos derivativos: "orientação para a comunidade", "centralidade na família" e "competência cultural" (Starfield, 2002). Esses sete atributos foram caracterizados por Lima (2016) além de correlacionados com as variáveis do PMAQ.

Starfield et al. (2005) demonstram que sistemas de saúde com APS forte estão associados a melhores resultados de saúde. Mostrou que em países industrializados com orientação para a
APS, observou-se a relação de uma APS robusta com menores taxas de mortalidade por qualquer causa (por exemplo câncer, doença cardiovascular, doenças respiratórias, mortalidade prematura e infantil), além de aumento da expectativa de vida e melhoria na autopercepção de saúde dos usuários.

Barbara Starfield e colegas (Cassady et al., 2000) sintetizaram os sete atributos em um Instrumento de Avaliação da Atenção Primária (Primary Care Assessment Tool - PCATool), posteriormente adaptados, traduzidos e validados no Brasil (Harzheim et al., 2006; Macincko \& Almeida, 2004). Porém, são incipientes os estudos aplicados do PCATool no SUS (D'Avila et al., 2017).

Em 2011, no bojo da nova PNAB, o Ministério da Saúde lançou o Programa de Avaliação para a Qualificação do Sistema Único de Saúde (SUS) (Brasil, 2011, 2012, 2015), instituindo o instrumento de Autoavaliação para a Melhoria do Acesso e da Qualidade da Atenção Básica (AMAQ) (Brasil, 2012). Nesse escopo, o PMAQ, que teve três ciclos (2012, 2014 e 2015/2017), tem possibilitado um melhor entendimento da estrutura e forma de organização da ESF, sendo 
um banco de dados importante neste aspecto. Entretanto, as avaliações feitas, não permitem, por si só, a avaliação da ampliado para outras unidades de atenção primária de saúde, mas, no 3o. ciclo do PMAQ (2015/2017), contou pela primeira vez com um quadro de indicadores de resultados das unidades (Brasil, 2017a, 2017b). Dessa forma, Lima (2016: 64) destaca o programa PMAQ-AB, do Departamento de Atenção Básica do Ministério da Saúde, enquanto estratégia institucionalizada de avaliação da atenção básica do SUS.

Em 2019, o MS estruturou o Programa Previne Brasil (Brasil, 2019). Herzheim (2020) alega a radicalização dos atributos da APS, o financiamento por capitação ponderada para induzir a vinculação dos usuários da APS para indução à responsabilização dos gestores e dos profissionais pelas pessoas que assistem, além da definição de carteira de serviços da APS. O Previne Brasil é focado em resultados na avaliação da APS por meio de três macro indicadores: o Net Promoter Score (NPS)1 mede afiliação, fidelização e confiança (longitudinalidade) entre as pessoas e as equipes de APS, O PDRQ-9 (Wollmann et al., 2018) - mede a qualidade e a confiança da relação entre as pessoas e o médico de família e comunidade (longitudinalidade). E o PCATOOLBrasil - mede a presença e a extensão dos quatro atributos essenciais (acesso de primeiro contato, longitudinalidade, integralidade e coordenação), além de dois atributos derivados (orientação comunidade e centralidade na família) (Harzheim, 2020). Apresenta apenas sete indicadores da clínica/epidemiologia para o "Pagamento por Desempenho", relativos às ações estratégicas de saúde da mulher, prénatal, saúde da criança e hipertensão arterial e diabetes mellitus para 2020/2021, projetando atingir 21 indicadores até 2022.

Segundo (Lima, 2016) o Instrumento PCATool mede a presença e a extensão dos 4 atributos essenciais e dos 3 atributos derivados da APS, baseando-se em um modelo de avaliação da qualidade de serviços de saúde, de acordo com aspectos de estrutura, processo e resultados de Donabedian. As variáveis dos três ciclos do PMAQ também estão voltadas para esse tripé ou para algum aspecto normativo. O Previne Brasil pretende medir aspectos de resultados e desempenho das equipes da APS. No entanto, nenhum desses instrumentos e programas aborda os aspectos de mensuração de impactos e efeitos comparativos na população assistida.

\section{Avaliação de impacto na APS}

A avaliação do impacto através de estudos de políticas públicas baseado em evidências (De Faria, 2005) pode apoiar os gestores nos possíveis planejamentos e decisões estratégicas dos sistemas de saúde, por exemplo, com estudos comparativos de eficácia, efetividade e impacto de médio e longo prazos.

No que se refere à efetividade, a Joint Comission on Accreditation of Healthcare Organizations (JCAHO, 1993) a define como o grau em que uma determinada intervenção ou tecnologia médica traz benefícios para indivíduos de uma população definida, sob condições regulares de uso. Assim, Ugá et al. (2013) afirmam que a efetividade pode ser mensurada a partir dos resultados da intervenção do sistema de serviços sobre a saúde da população.

Por sua vez, reunindo-se os diferentes enfoques é possível dizer que a resolutividade envolve aspectos relativos à demanda, à satisfação do cliente, às tecnologias dos serviços de saúde, à existência de um sistema de referência preestabelecido, à acessibilidade dos serviços, à formação dos recursos humanos, às necessidades de saúde da população, à adesão ao tratamento, aos aspectos culturais e socioeconômicos da clientela, entre outros (Turrini et al., 2008).

Diante das limitações dos modelos de medidas unidimensionais da eficácia ou performance organizacional (Brunet et al., 1991 apud Carvalho, 2007: 164) prosperou variados

\footnotetext{
${ }^{1}$ https://endeavor.org.br/estrategia-egestao/nps/
} 
modelos operacionais multidimensionais, sendo os principais caracterizados por Carvalho (2007). Assim, pode-se explorar os diversos indicadores socioeconômicos, os quais condicionam aspectos epidemiológicos da população, bem como direcionam a política pública em saúde.

Propõem-se que os indicadores sociodemográficos e econômicos podem ser disponibilizados em mapas temáticos, os quais permitem uma visualização espacial das condições socioeconômicas dos municípios. A análise de correlação permitirá observar o grau de associação entre indicadores socioeconômicos, ou entre os indicadores e variáveis de saúde. Por sua vez, a análise exploratória de dados espaciais permite observar como as condições socioeconômicas dos municípios se relacionam com as condições socioeconômicas ou de saúde de seus vizinhos, bem como a identificação de clusters espaciais. Ademais, é possível empregar análise de componentes principais se o objetivo for o de transformar um conjunto de indicadores socioeconômicos em um único índice. Já a análise de regressão múltipla permitirá observar como as condições socioeconômicas afetam determinadas variáveis de saúde, estabelecendo potenciais relações de causalidade. Por fim, o conjunto de indicadores socioeconômicos podem ser utilizados na estimação de uma função de produção em saúde, ajudando a mensurar a eficiência técnica em saúde através da análise de input-output.

A partir de tais informações coletadas propõemse executar uma Análise Exploratória de Dados Espaciais (AEDE) visando descobrir padrões de associação espacial (clusters espaciais) e sugerir diferentes regimes espaciais e outras formas de instabilidade espacial (Anselin, 1988, 1995).

Em seguida, pode-se usar o Diagrama de dispersão de Moran que permite visualizar a autocorrelação espacial, e mostra a defasagem espacial da variável de interesse no eixo vertical e o valor da variável no eixo horizontal, além de padrões de associação local espacial entre as áreas e suas regiões vizinhas (Almeida, 2012).

Há a opção de usar a Análise Envoltória de Dados (Data Envelopment Analysis - DEA), técnica não- paramétrica que emprega programação matemática para construir fronteiras de eficiência por unidades tomadoras de decisões (Decision Making Units - DMUs), através da análise multicritério da eficiência, pelos indicadores críticos de desempenho e fronteiras comparativas de performances das DMUs. O DEA compara um certo número de DMUs que realizam tarefas similares e se diferenciam unicamente nas quantidades dos recursos consumidos e das saídas produzidas.

Por fim, pode-se aplicar a regressão segmentada de dados de séries temporais, para analisar como e se as unidades de saúde, por modelo de gestão, estão produzindo efeitos sobre os indivíduos e/ou famílias atendidas no território, e em que extensão estes efeitos podem ser atribuíveis à intervenção. Esta etapa analítica do projeto de pesquisa tem como escopo estabelecer se a adoção de um modelo privado (OSs) de gestão dos serviços públicos de saúde teve impactos positivos sobre a eficácia na prestação de serviços de saúde nos municípios estudados em diferentes segmentos temporais comparadamente com municípios controle. Nesta etapa, a metodologia utilizada para analisar o impacto será a regressão segmentada de dados de séries temporais, conhecida como Interrupted Time Series Analysis (ITSA) ou análise de séries temporais interrompidas (ITS) (Mcdowall et al., 2019: 17). A análise de séries temporais interrompidas é um dos desenhos quase experimentais mais forte para avaliar os efeitos longitudinais de tais intervenções delimitadas pelo tempo (Cook \& Campbell, 1979; Gillings et al., 1981; Wagner et al., 2002) e, permitem avaliar, em termos estatísticos, quanto uma intervenção mudou um resultado de interesse, imediatamente e ao longo do tempo e se outros fatores além da intervenção poderiam explicar a mudança, tendo sido usado para avaliar diversos aspectos do programa Farmácia Popular (Silva et al., 2017).

\section{CONCLUSÕES}

No foco da proposta desse trabalho, um estudo científico (Ramos \& De Seta, 2019) que 
comprovou que as unidades próprias da prefeituras são mais eficientes que OSs na gestão de UBS em com os seguintes destaques: "As Prefeituras de Belo Horizonte e Vitoria apresentam melhores resultados ao administrar diretamente a saúde básica em suas cidades do que as organizações sociais de saúde (OSs) contratadas para fazer o mesmo nos municípios de São Paulo e Rio de Janeiro.

Na presente proposta pretende-se alcancar os seguintes resultados: Mapeamento de dados e informações sociodemográficas, econômicas e seus potenciais reflexos epidemiológicos para a caracterização técnica e o georeferenciamento das áreas de abrangências das UBS de Fortaleza e Sobral; Estabelecimento de padrões de influências (clusters espaciais) das unidades, por meio de cálculos de autocorrelação e associação espacial, para a estimação de uma função de produção em saúde das UBS, ajudando a mensurar a eficiência técnica em saúde através da análise de input-output; Mapeamento de dados e informações de produtividade, eficiência, de efetividade e resolutividade do atendimento registrados nas UBS de Fortaleza e Sobralsubsidiando os cálculos de indicadores multidimensionais de saúde; Avaliação da produtividade e da eficiência atendimento das UBS com diferentes regimes de modelos de gestão de serviços de saúde (Fortaleza antes e depois das mudanças comparadamente ao município de Sobral; Estudo sobre o impacto do financiamento e modelos de gestão na eficiência dos serviços de APS dos últimos anos em relação à contribuição para o avanço e a melhoria do serviço nas unidades de APS de Fortaleza e Sobral; Consolidação de um instrumento/escala de medida de efetividade e resolutividade (qualidade), na percepção e experiência dos usuários dos serviços das UBS de Fortaleza comparativa às de Sobral, aplicáveis aos outros serviços e unidades do SUS e; por fim, Esclarecimento com evidência científica robusta sobre a análise do melhor regime de financiamento e gestão da APS (Fortaleza antes e depois das mudanças, comparadamente outras metrópoles selecionadas), quanto à eficiência, ao impacto, à efetividade e à resolutividade (qualidade) nas UBS com diferentes regimes de modelos de gestão de serviços de saúde e, adaptáveis a outras realidades do SUS.

Dessa forma, ancorados em uma base teóricometodológica robusta pode-se validar metodologias e instrumentos capazes de subsidiar a análise de impacto, efetividade e resultados em contextos comparáveis da APS para apoio à tomada de decisão no SUS. 


\section{REFERÊNCIAS}

Albuquerque, C., \& Martins, M. (2017). Indicadores de desempenho no Sistema Único de Saúde: uma avaliação dos avanços e lacunas. Saúde em Debate, 41(Especial), 27. https://doi.org/10.1590/0103-11042017S10

Alfradique, M. E., Bonolo, P. F., Dourado, I., Lima-Costa, M. F., Macinko, J., Mendonça, ... Turci, M. A. (2009). Internações por condições sensíveis à atenção primária: a construção da lista brasileira como ferramenta para medir o desempenho dosistema de saúde (Projeto ICSAP-Brasil). Caderno de Saúde Pública, 25(6), 1337-1349.

Almeida, E. (2012). Econometria Espacial Aplicada (E. Alínea, Ed.).

Almeida, P. F., \& Giovanella, L. (2008a). Avaliação em Atenção Básica à Saúde no Brasil: mapeamento e análise das pesquisas realizadas e/ou financiadas pelo Ministério da Saúde entre os anos de 2000 e 2006. Cad. Saúde Pública, 24(8), 16.

Almeida, P. F., \& Giovanella, L. (2008b). Avaliação em Atenção Básica à Saúde no Brasil: mapeamento e análise das pesquisas realizadas e/ou financiadas pelo Ministério da Saúde entre os anos de 2000 e 2006. Cad. Saúde Pública, 24(8), 16.

Andrade, L. O. M., \& Martins Júnior, T. (1999). Saúde da Família: Construindo um novo modelo A experiência de Sobral. Sanare - Revista Sobralense de Políticas Públicas, 1(1), 7-17.

Anselin, L. (1988). Spatial Econometrics: Methods and Models. Studies in Operational Regional Science (K. Academic, Ed.).

Anselin, L. (1995). Local Indicators of Spatial Association - LISA. Geographical Analysis, 27(2), 93115.

Arretche, M. (2003). Financiamento federal e gestão local de políticas sociais: o difícil equilíbrio entre regulação, responsabilidade e autonomia. Revista Ciência e Saúde Coletiva, 8(2), 331-345.

Avila, M. M. M. (2011). O Programa de Agentes Comunitários de Saúde no Ceará: o caso de Uruburetama Ciênc. saúde coletiva, 16(1), 349-360.

Barreto, M. L., Rasella, D., Machado, D. B., Aquino, R., Lima, D., Garcia, L. P., . . Travassos, C. (2014). Monitoring and evaluating progress towards Universal Health Coverage in Brazil. PLoS Med, 11(9). https://doi.org/10.1371/journal.pmed.1001692

Barreto, M. L., Teixeira, M. G., Bastos, F. I., Ximenes, R. A. A., Barata, R. B., \& Rodrigues, L. C. (2011). Sucessos e fracassos no controle de doenças infecciosas no Brasil: o contexto social e ambiental, políticas, intervenções e necessidades de pesquisa. The Lancet.

Bogdan, R., \& Biklen, S. (2010). Investigação qualitativa em educação: uma introdução à teoria e aos métodos (Porto, Ed.).

Bosi, M. L. M., \& Uchimura, K. Y. (2007). Avaliação da qualidade ou avaliação qualitativa do cuidado em saúde? Revista de Saúde Pública, 41(1), 150-153.

Brasil. (1995). Plano Diretor da Reforma do Aparelho do Estado (PDRAE), conduzido pelo então Ministro da Administração Federal. M. d. d. F. e. R. d. Estado. http://www.biblioteca.presidencia.gov.br/publicacoes-oficiais/catalogo/fhc/plano-diretor-dareforma-do-aparelho-do-estado-1995.pdf 
Brasil. (1997). Saúde da Família: uma estratégia para a reorientação do modelo assistencial. S. d. A. à. Saúde. http://bvsms.saude.gov.br/bvs/publicacoes/cd09 16.pdf.

Brasil. (2000). Estratégia Saúde da Família: Cadernos de Atenção Básica. S. d. A. à. S. D. d. A. Básica.

http://bvsms.saude.gov.br/bvs/publicacoes/implantacao_unidade saude_familia_cab1.pdf

Brasil. (2006). Política nacional de atenção básica. S. d. A. à. S. D. d. A. Básica.

Brasil. (2009). Diretrizes do NASF ((Caderno de Atenção Básica n. 27), Issue. S. d. A. à. S. D. d. A. Básica.

Portaria no 2488 de 21 de outubro de 2011. Aprova a Política Nacional de Atenção Básica, estabelecendo a revisão de diretrizes e normas para a organização da atenção básica, para a Estratégia Saúde da Família (ESF) e o Programa de Agentes Comunitários de Saúde (PACS), (2011).

Brasil. (2012). Autoavaliação para a Melhoria do Acesso e da Qualidade da Atenção Básica: AMAQ. S. d. A. à. S. D. d. A. Básica.

Brasil. (2015). Alternativas de Gerência de Unidades Públicas de Saúde (978-85-8071-029-8).

Brasil. (2016). O SUS em vista: indicadores socioeconômicos, de saúde, de financiamento e de serviços de saúde. Ministério da Saúde

Brasil. (2017a). Autoavaliação para melhoria do acesso e da qualidade da atenção básica: AMAQ. Brasília: Ministério da Saúde, Secretaria de Atenção à Saúde, Departamento de Atenção Básica Retrieved from http://189.28.128.100/dab/docs/portaldab/publicacoes/amaq_2017.pdf

Brasil. (2017b). Manual instrutivo para as equipes de atenção básica e NASF - Programa Nacional de Melhoria do Acesso e da Qualidade da Atenção Básica (PMAQ) - Terceiro ciclo - (2015-2017). Brasília: Ministério da Saúde, Secretaria de Atenção à Saúde, Departamento de Atenção Básica Retrieved from http://189.28.128.100/dab/docs/portaldab/documentos/Manual Instrutivo 3 Ciclo PMAQ.pd f

Portaria no 2.979, de 12 de novembro de 2019, (2019).

Brunet, L., Brassard, A., \& Corriveau, L. (1991). Administration Scolaire et efficacité dans le organizations (É. A. DÁRC, Ed.). Éditions Agence DÁRC.

Cabral, A. C. (2019). Legislativo aprova matéria sobre organizações sociais em $1^{a}$ discussão http://www.cmfor.ce.gov.br/legislativo-aprova-materia-sobre-organizacoes-sociais-em-1adiscussao/

Carvalho, C. (2007). Organizações, actores envolvidos e partes interessadas: Determinantes da saliência dos stakeholders e sua relação com o desempenho organizacional [Doctoral, Universidade de Coimbra]. Coimbra, Portugal.

Cassady, C. E., Starfield, B., Hurtado, M. P., Berk, R. A., Nanda, J. P., \& Friedenberg, L. A. (2000). Measuring consumer experiences with Primary Care Pediatrics, 105(4 pt 2), 6.

Portaria no 232/92, de 15 de maio de 1992. Estabelece normas técnicas e operacionais de planejamento, execução, acompanhamento e avaliação do Programa de Agentes de Saúde, criado pelo Decreto Estadual no 19.945, de 02 de janeiro de 1989, e dá outras providências, 
(1992).

Ceará. (2019a). Panorama estadual da atenção Primária à saúde. http://189.28.128.100/dab/docs/portaldab/documentos/apresentacoes/panorama_estadual_a ps.pdf

Ceará. (2019b). Relatório Panorama Estadual Atenção Primária à Saúde. http://189.28.128.100/dab/docs/portaldab/documentos/apresentacoes/panorama_estadual_a ps.pdf

Cook, T. D., \& Campbell, D. T. (1979). Quasi-experimentation. Design \& analysis issues for field settings (H. M. Company, Ed.).

De Faria, C. A. P. (2005). A política da avaliação de políticas públicas. Revista Brasileira de Ciências Sociais, 20(59), 14.

De Lima, E. (2013). MPF pede que polícia federal investigue contrato da gestão... . Caderno Blogs e Coluna.

Dourado, I., Oliveira, V. B., Aquino, R., Bonolo, P., Lima-Costa, M. F., Medina, M. G., ... Macinko, J. (2011). Trends in primary health care-sensitive conditions in Brazil: the role of the Family Health Program (Project ICSAP-Brazil). Med Care, 49(6), 577-584.

D’Avila, O. P., Pinto, L. F. S., Hauser, L., Gonçalves, M. R., \& Harzheim, E. (2017). O uso do Primary Care Assessment Tool (PCAT): uma revisão integrativa e proposta de atualização. Ciênc. \& Saúde Coletiva, 22(3), 11. https://doi.org/10.1590/1413-81232017223.03312016

Flick, U. (2009). Introdução à pesquisa qualitativa (Artmed, Ed. 3a ed.). Artmed.

Fortaleza. (2019). Relatório de Prestação de Contas de Governo. Balanço Geral https://transparencia.fortaleza.ce.gov.br/index.php/contasPublicas/exibirBalancoGeral

Fortaleza. (2020). Plano Municipal de Contingência para Enfrentamento da Infecção Humana pelo Novo Coronavírus SARS-CoV-2

Gillings, D., Makuc, D., \& Siegel, E. (1981). Analysis of interrupted time series mortality trends: an example to evaluate regionalized perinatal care. American Journal of Public Health, 71, 38-46.

Giovanella, L., \& Mendonça, M. H. M. (2012). Atenção primária à saúde. In L. Giovanella, S. Escorel, \& L. V. C. Lobato (Eds.), Políticas e Sistema de saúde no Brasil (2a ed., pp. 575-626). Fiocruz.

Giovanella, L., Ruiz, G., Feo, O., Tobar, S., \& Faria, M. (2012). Sistemas de Salud en América del Sur. In Sistemas de Salud en Suramérica: desafios para la universidad, la integralidad y la equidad. ISAGS.

Gragnolati, M., Lindelow, M., \& Couttolenc, B. (2013). Twenty Years of Health System Reform in Brazil: An Assessment of the Sistema Único de Saúde. Directions in Development (W. Bank, Ed.). https://doi.org/10.1596/978-0-8213-9843-2

Harzheim, E. (2020). "Previne brasil": bases da reforma da atenção primária à saúde Ciênc. \& Saúde Coletiva, 25(4), 7. https://doi.org/10.1590/1413-81232020254.01552020

Harzheim, E., Starfield, B., Rajmil, L., Dardet, C. A., \& Stein, A. T. (2006). Internal consistency 
and reliability of Primary Care Assessment Tool (PCATool-Brasil) for child health services Cadernos de Saúde Pública, 22(8), 11.

JCAHO, J. C. O. A. O. H. C. O.-. (1993). The measuring mandate - on the road to performance improvement in health care. Department of Publications.

Lima, J. G. (2016). Atributos da Atenção Primária nas regiões de saúde: uma análise dos dados do Programa Nacional de Melhoria do Acesso e da Qualidade da Atenção Básica FIOCRUZ]. Rio de Janeiro

Macedo, F. (2018). A verdade sobre as OSS em saúde. Caderno de Política.

Macincko, J., \& Almeida, C. (2004). Organization and delivery of primary health care services in Petropolis, Brazil. International Journal of Health Planning and Management, 4(19), 15.

Macinko, J., Sousa, M. F. M., Guanais, F., \& Simões, C. S. (2007). Going to scale with communitybased primary care: an analysis of the family health program and infant mortality in Brazil, 19992004. Soc Sci Med, 65, 2070-2080.

Mcdowall, D., Mccleary, R., \& Bartos, B. J. (2019). Interrupted time series analysis. In: Oxford University Press.

Moita, G. F. (2021). As bases teóricos-conceituais ( framework ) de avaliação de desempenho e resultados aplicado nas instituições de saúde no Brasil [The theoretical-conceptual bases (framework) of performance evaluation and results applied in health institutions in Brazil] (a. $y$. t. g. The creation of the Unified Health System (SUS) in Brazil in 1988 represented advances in the systemic organization and decentralization of the unified management however, Trans.). In M. A. Fernandes (Ed.), Gestão Organizacional: realidade atual e perspectivas futuras (pp. 119-145). Synapse. https://doi.org/10.36599/editpa-2021 gorap-009

Moita, G. F., Hartz, Z. M. A., Melanda, V. S., Colares, G. M., \& Kaminski, A. P. R. (2021). Controlo Social Tutelado? Enfrentamentos e dinâmicas democráticas na implantação de organizações sociais na saúde de capital do Nordeste do Brasil [Protected social control? Confrontations and democratic dynamics in the implementation of social organizations in the health capital of northeastern Brazil]. Anais do Instituto de Higiene e Medicina Tropical, 20(2021), 12.

Moita, G. F., Raposo, V. M. R., \& Barbosa, A. C. Q. (2019). Validação colaborativa de macrodimensões e indicadores-chave para avaliação de performance de serviços de saúde no Brasil [Collaborative validation of macrodimensions and key indicators for health services performance evaluation in Brazil]. Rev. Saúde em Debate, 43(5), 232-247. https://doi.org/10.1590/0103-11042019s519

Ramos, A. L. P., \& De Seta, M. H. (2019). Atenção primária à saúde e Organizações Sociais nas capitais da Região Sudeste do Brasil: 2009 e 2014. Cad. Saúde Pública, 35(4).

Raposo, V. (2007). Governação hospitalar - uma proposta conceptual e metodológica para o caso português Universidade de Coimbra]. Coimbra.

Rasella, D., Aquino, R., \& Barreto, M. L. (2010). Reducing childhood mortality from diarrhea and lower respiratory tract infections in Brazil. Pediatrics, 126, 534-540. https://doi.org/10.1542/peds.2009-3197

Rasella, D., Harhay, M. O., Pamponet, M. L., Aquino, R., \& Barreto, M. L. (2014). Impact of primary health care on mortality from heart and cerebrovascular diseases in Brazil: a nationwide analysis 
of longitudinaldata. BMJ, 349. https://doi.org/10.1136/bmj.g4014

Ribeiro, M. A., \& Cunha, C. G. (2018). Atenção Primária à Saúde de Sobral. Relatório de Planejamento e Avaliação da Coordenadoria de Vigilância do Sistema de Saúde de Sobral

Sancho, L. G., \& Dain, S. (2012). Avaliação em Saúde e Avaliação Econômica em Saúde: introdução ao debate sobre seus pontos de interseção. Ciência e Saúde Coletiva, 17, 765-774.

Santana, R., \& Costa, C. (2008). A integração vertical de cuidados de saúde: aspectos conceptuais e organizacionais Revista Portuguesa de Saúde Pública, 7, 28.

Santana, R. M. C. (2011). O financiamento por capitação ajustada pelo risco em contexto de integração vertical de cuidados de saúde: a utilização dos consumos com medicamentos como proxy da carga de doença em ambulatório [Doctoral, Universidade Nova de Lisboa]. Lisboa.

Saúde, M. d. (2017). Nova política da Atenção Básica amplia acompanhamento domiciliar a mais de 5 milhões de hipertensos e diabéticos https://www.saude.gov.br/noticias/agenciasaude/29706-nova-politica-da-atencao-basica-amplia-acompanhamento-domiciliar-a-mais-de5-milhoes-de-hipertensos-e-diabeticos

Silva, R. M. D., Chaves, G. C., Chaves, L. A., Campos, M. R., Luiza, V. L., Bertoldi, A. D., . . Emmerick, I. C. M. (2017). Farmácia Popular Program: pharmaceutical market analysis of antihypertensive acting on the renin-angiotensin system medicines. Ciencia \& Saude Coletiva, 22, 2501-2512.

Starfield, B. (2002). Atenção Primária: equilíbrio entre necessidades de saúde, serviços $e$ tecnologia (E. brasileira, Ed.). UNESCO-Organização das Nações Unidas para a Educação, a Ciência e a Cultura / Ministério da Saúde.

Starfield, B., Shi, L., \& Macincko, J. (2005). Contribution of primary care to health systems and health. Journal List Milbank Q, 83(3), 46.

Stein, A. T., Harzheim, E., Costa, M., Busnello, E., Rodrigues, \& C, L. (2002). The relevance of continuity of care to the chaos in the emergency services in Brazil. Family Pract, 19(2), 207-210.

Thomas, J. R., \& Nelson, J. K. (2002). Métodos de pesquisa em atividade física (Artmed, Ed. 3a ed.).

Turrini, R. N. T., Lebrão, M. L., \& Galvão Cesar, C. L. (2008). Resolutividade dos serviços de saúde por inquérito domiciliar: percepção do usuário. Cadernos de Saúde Pública, 24, 663-674.

Ugá, M. A. D., Laguardia, J., \& Porto, S. M. (2013). A efetividade do sistema de saúde brasileiro: uma avaliação através de indicadores referentes a condições evitáveis. Rev. Eletr. de Com. Inf. Inov. Saúde, 7(2), 1-15.

Viana, K. C. A. (2010). As Organizações Sociais no Âmbito do Governo do Estado do Ceará: Celebração, Acompanhamento e Fiscalização dos Contratos Congresso Consad de Gestão Pública, Brasília. https://docplayer.com.br/16626546-As-organizacoes-sociais-no-ambito-do-governodo-estado-do-ceara-celebracao.html

Victora, C. V. (2011). Series from the Lancet journals (Brazil). The Lancet.

Vieira, F. S., \& Zucchi, P. (2013). Financiamento da assistência farmacêutica no sistema único de saúde. Saúde e Sociedade, 22(1), 12. https://doi.org/10.1590/S0104-12902013000100008 
Volpato, L. F. (2014). Utilização de uma ferramenta para análise da qualidade nos serviços públicos de saúde [Doctoral, Universidade estadual de campinas]. Piracicaba

Volpato, L. F., Meneghim, M. C., Pereira, A. C., \& Ambrosano, G. M. B. (2010). Planejamento da qualidade nas unidades de saúde da família, utilizando o Desdobramento da Função Qualidade (QFD). Cad. Saúde Pública, 26(8), 1561-1572.

Wagner, A. K., Soumerai, S. B., Zhang, F., \& Ross-Degnan, D. (2002). Segmented regression analysis of interrupted time series studies in medication use research. Journal of clinical pharmacy and therapeutics, 27(4), 299-309.

Wollmann, L., Hauser, L., Mengue, S. S., Agostinho, M. R., Roman, R., Feltz-Cornelis, C. M. V. D., \& Harzheim, E. (2018). Crosscultural adaptation of the Patient-Doctor Relationship Questionnaire (PDRQ-9) in Brazil Rev. Saúde Pública, 10. https://doi.org/10.11606/S1518$\underline{8787.2018052000380}$

Yin, R. K. (2010). Estudo de caso: planejamento e métodos (Bookman, Ed. 4a ed.). 